\title{
A nomogram for the prediction of renal outcomes among patients with idiopathic membranous nephropathy
}

\author{
JI ZHANG ${ }^{1}$, SHUFANG PAN ${ }^{2}$, DUO LI ${ }^{1}$, ZHANYUAN LI ${ }^{2}$, LINGWEI JIN ${ }^{2}$, ZHIHONG ZHOU $^{2}$ and MIN PAN ${ }^{2}$ \\ ${ }^{1}$ Department of Nephrology, The First Affiliated Hospital of Wenzhou Medical University; \\ ${ }^{2}$ Department of Nephrology, The Second Affiliated Hospital and Yuying Children's Hospital \\ of Wenzhou Medical University, Wenzhou, Zhejiang 325000, P.R. China
}

Received March 3, 2020; Accepted July 1, 2020

DOI: $10.3892 /$ etm.2020.9063

\begin{abstract}
The early prediction of renal outcomes in patients with idiopathic membranous nephropathy (iMN) remains challenging. The present retrospective study evaluated patients with iMN confirmed by renal biopsy. An optimized Cox regression model and a nomogram were constructed for the early prediction of renal outcomes. A total of 141 patients who met the inclusion criteria were evaluated in the present study. In total $18(12.8 \%)$ patients eventually progressed to the endpoint, 6 of whom developed end-stage renal disease, and one patient died during follow-up. The optimized model demonstrated that 24-h proteinuria [hazard ratio (HR) 1.24; 95\% CI, 1.10-1.40; P-value <0.001] and chronic tubulointerstitial injury [referred to as grade 0 , grade 1 (HR), 5.12; $95 \%$ CI, 1.33-19.75; P-value $=0.02$ ] or grade $2(\mathrm{HR}, 6.43 ; 95 \% \mathrm{CI}$, 1.35-30.59; P-value=0.02) were independent risk factors for
\end{abstract}

Correspondence to: Dr Min Pan, Department of Nephrology, The Second Affiliated Hospital and Yuying Children's Hospital of Wenzhou Medical University, 109 Xueyuan Road, Wenzhou, Zhejiang 325000, P.R. China

E-mail:pm_1234@163.com

Abbreviations: iMN, idiopathic membranous nephropathy; HR, hazard ratio; ETR, estimated three-year renal survival rate; $\mathrm{MN}$, membranous nephropathy; PLA2R, M-type phospholipase A2 receptor; eGFR, estimated glomerular filtration rate; CTX, cyclophosphamide; CKD, chronic kidney disease; CKD-EPI, chronic kidney disease epidemiology collaboration equation; $\mathrm{BP}$, blood pressure; UPCR, urine protein-to-creatinine ratio; AIC, Akaike's information criterion; ROC, receiver operating characteristic; AUCs, areas under the ROC curve; SD, standard deviation; IQRs, interquartile ranges; ESRD, end-stage renal disease; ACEI, angiotensin-converting enzyme inhibitor; ARB, angiotensin receptor blocker; $\mathrm{CI}$, confidence interval; HDL-C, high-density lipoprotein cholesterol; LDL-C, low-density lipoprotein cholesterol; SBP, systolic blood pressure; DBP, diastolic blood pressure; CR, complete remission; PR, partial remission; NR, no remission.

Key words: idiopathic membranous nephropathy, prognostic model, renal outcomes, chronic kidney disease a poor renal outcome. Patients with an estimated three-year renal survival rate (ETR) less than 0.87 had a high risk of a poor renal outcome. In addition, patients with an ETR of 0.87 to 0.98 more quickly developed a decreased estimated glomerular filtration rate after two years of follow-up. In the present study a nomogram for the early prediction of renal outcomes in patients with iMN was developed. This nonogram suggested that patients with an ETR of 0.87-0.98 should receive greater attention during follow-up.

\section{Introduction}

Membranous nephropathy ( $\mathrm{MN}$ ) is considered an autoimmune disease and is characterized by autoimmune antibodies deposited on the glomerular basement membrane. It is a leading cause of nephrotic syndrome in adults $(1,2)$. Approximately $20 \%$ of MN cases are caused by systemic diseases or exposure, termed secondary $\mathrm{MN}$, while $80 \%$ of cases are located in the kidney and termed idiopathic MN (iMN) (2,3). Autoimmune antibodies against the $\mathrm{M}$-type phospholipase $\mathrm{A} 2$ receptor (PLA2R) have been detected in $70 \%$ of $\mathrm{MN}$ cases and against thrombospondin type 1 domain-containing 7A in 2-3\% of $\mathrm{MN}$ cases $(4,5)$. It is thought that iMN with a benign course toward spontaneous remission occurs in approximately 30-60\% of cases within the first 2 years after presentation, and mild progression of renal function occurs during follow-up (6-8). However, approximately $30-50 \%$ of iMN cases gradually progress to renal insufficiency within 5-10 years (9-11) and immunosuppressive treatment should be offered to these patients with a medium or high risk of renal progression (12). Unfortunately, the early identification of such medium- or high-risk patients remains elusive (13).

Cattran et al and Pei et al $(14,15)$ developed a logistic model called the Toronto Risk Score for the classification of patients with iMN, but the calculation process is complicated as the variables included in the model require conversion and follow-up data for at least 6 months. Significant differences in clinical variables at the time of renal biopsy have been reported between iMN patients with different renal outcomes $(6,16-18)$. Thus, an early predictive model based on these variables may be warranted. The present study was designed to construct a simple and convenient predictive model to facilitate early prediction of the renal outcomes of iMN patients. 


\section{Materials and methods}

Subjects. Patients who received a biopsy-based diagnosis of iMN between January 2010 and December 2018 at the Second Affiliated Hospital of Wenzhou Medical University were included in this retrospective analysis. The inclusion criteria were as follows: i) Age $>18$ years; ii) an estimated glomerular filtration rate $(\mathrm{eGFR})>15 \mathrm{ml} / \mathrm{min} / 1.73 \mathrm{~m}^{2}$ at renal biopsy; and iii) a follow-up time $>12$ months. The exclusion criteria were: i) Secondary membranous nephropathy, such as hepatitis B-associated membranous nephropathy or systemic lupus erythematosus; and ii) the presence of malignant tumors.

Collection of clinical and laboratory data. Patient demographic data and blood pressure were recorded at the time of renal biopsy. Clinical laboratory tests, including the results of serum biochemical tests and urinary tests, were also collected at the time of renal biopsy and during the follow-up. At the time of renal biopsy, $24 \mathrm{~h}$ proteinuria was assessed and the spot urinary protein-to-creatinine ratio was determined during the follow-up. Serum anti-PLA2R antibody levels were determined using the commercial ELISA kits (EUROIMMUN AG; cat. no. EA 1254-9601 G) following the standard instruction (19). The treatment strategy was decided based on the Kidney Disease Improving Global Outcomes guidelines (20). However, immunosuppressive therapy was started more rapidly among patients with obviously reduced levels of serum albumin ( $<25 \mathrm{~g} / \mathrm{l}$; normal range, 35.0-55.0 g/l) combined with a nephritic range of proteinuria ( $>4 \mathrm{~g}$ per day; normal range, $0.0-0.15 \mathrm{~g}$ per day) or gradually increased serum creatinine in our cohort. Patients that received different immunosuppressive treatment regimens were divided into four groups: i) No, did not receive any immunosuppressants or received only corticosteroids; ii) cyclophosphamide (CTX), received CTX with corticosteroids; iii) $\mathrm{CNI}$, received tacrolimus or cyclosporine with/without corticosteroids, and iv) others, received multiple immunosuppressants or immunosuppressants other than the above. The eGFR was evaluated using the chronic kidney disease (CKD) epidemiology collaboration (CKD-EPI) equation (21). Hypertension was defined as a blood pressure (BP) $>140 / 90 \mathrm{mmHg}$ (22) or a diagnosis of hypertension before the renal biopsy. Complete remission was defined as a spot urine protein-to-creatinine ratio (UPCR) $<0.2 \mathrm{~g} / \mathrm{g}$ and serum albumin $>35 \mathrm{~g} / 1$. Partial remission was defined as a spot UPCR $>0.2 \mathrm{~g} / \mathrm{g}$ and $<3.5 \mathrm{~g} / \mathrm{g}$ and serum albumin $>30 \mathrm{~g} / \mathrm{l}$. Patients who did not meet the above criteria were defined as having no remission. Relapse was defined as the recurrence of nephrotic syndrome in patients with partial or complete remission.

Renal histopathology. Kidney tissues were obtained from all patients during routine renal biopsies. Specimens from all patients were allocated and processed according to standard techniques for light microscopy, immunofluorescence microscopy and electron microscopy (23). Membranous lesions from all iMN cases were classified (stages I-IV) by electron microscopy based on the criteria of Ehrenreich and Churg (24). Chronic tubulointerstitial fibrosis was assessed using the Oxford classification of tubular atrophy/interstitial fibrosis and graded as grade 0 (absent to mild, $0-24 \%$ ), grade 1 (moderate, $25-49 \%$ ), or grade 2 (severe, $>50 \%$ ) (25). All renal biopsies were reviewed and scored independently by two renal pathologists (MP and DL) that were not blinded to patient history.

Constructing and verifying the early predictive model. A univariate Cox proportional hazard model was built using the rms package ( $\mathrm{R}$ package version 5.1-1; https://CRAN.R-project.org/package=rms) (26) and survival package ( $\mathrm{R}$ package version 2.41-3; https://CRAN.R-project. org/package $=$ survival) (27) to investigate the relationship between poor renal outcomes and histopathological or clinical variables. Next, covariates with $\mathrm{P}$-values $<0.1$ in univariate Cox models were selected to build a multivariate Cox regression model. Akaike's information criterion (AIC) (28) was used to simplify and optimize the model. The proportional hazard assumption of each covariate in the model was tested using the Therneau-Grambsch method (29). Finally, a nomogram was developed, and the estimated three-year renal survival rate (ETR) was calculated based on the model. Harrell's concordance index (Harrell's C-index) of the models was calculated using the Hmisc package ( $\mathrm{R}$ package version 4.1-0; https://CRAN.R-project.org/package=Hmisc) (30), and internal validation was performed using the rms package ( $\mathrm{R}$ package version 5.1-1; https:/CRAN.R-project. org/package $=$ rms) (26). Receiver operating characteristic (ROC) curves and areas under the ROC curve (AUCs) were used to assess the prognostic efficiency of the ETR and traditional risk factors. Then, the appropriate cut-off values were calculated based on the ROC curve.

Statistical analysis. Numerical variables are presented as the mean and standard deviation (SDs) or as the median and interquartile range (IQR) and were compared using the Wilcoxon test. Categorical variables are presented as cases with percentages and were compared with the $\chi^{2}$ test. The primary endpoints were a poor renal outcome [defined as an eGFR decrease of at least $50 \%$ from the baseline level or progression to end-stage renal disease (ESRD) during the follow-up] or death caused by MN. Renal survival was defined as the absence of the primary endpoints during the follow-up. Differences in proteinuria and eGFR changes in the groups during the follow-up were compared using factorial analysis of variance and Tukey's range test. All reported P-values were two-tailed, and $\mathrm{P}<0.05$ was considered statistically significant. Mathematical analyses were performed using $\mathrm{R}$ (version 3.5.1) (31).

\section{Results}

Clinical characteristics of including cases. A total of 141 cases met the inclusion criteria and were enrolled in the present study. The rate of male-to-female was 1.4:1. Approximately $95.7 \%$ of the enrolled cases were at CKD stage 1 or 2 , and half of the cases had hypertension at the time of renal biopsy. The median follow-up time of our cohort was 30 (IQR, 21-45) months. During the follow-up, $115(81.6 \%)$ patients received corticosteroids, and $103(81.5 \%)$ patients received immunosuppressants other than corticosteroids. A total of $85.8 \%$ of the patients achieved partial or complete remission of nephrotic syndrome, and 23 (19\%) patients relapsed during the follow-up. Almost all patients received angiotensin-converting enzyme inhibitor (ACEI) and/or angiotensin receptor blocker (ARB) 
Table I. Clinical and laboratory characteristics at the time of renal biopsy and during follow-up.

A, Characteristics at time of biopsy

\begin{tabular}{|c|c|}
\hline Characteristic [units, figure reported] & Value \\
\hline No. of cases & 141 \\
\hline Female $[\mathrm{n}(\%)]$ & $60(42.6)$ \\
\hline Age [years, mean (SD)] & $51.5(14.1)$ \\
\hline Serum albumin $[\mathrm{g} / 1$, mean $(\mathrm{SD})]$ & $24.2(5.4)$ \\
\hline Total cholesterol [mmol/1, mean (SD)] & $7.8(2.2)$ \\
\hline Triglycerides [mmol/1, mean (SD)] & $2.8(1.3)$ \\
\hline HDL-C [mmol/1, mean (SD)] & $1.5(0.5)$ \\
\hline LDL-C (mmol/l, mean (SD)] & $4.5(1.7)$ \\
\hline Serum uric acid [mg/dl, mean (SD)] & $6.3(1.4)$ \\
\hline Serum creatinine $[\mathrm{mg} / \mathrm{dl}$, median (IQR)] & $0.8(0.6-0.9)$ \\
\hline eGFR $\left[\mathrm{ml} / \mathrm{min} / 1.73 \mathrm{~m}^{2}\right.$, mean $\left.(\mathrm{SD})\right]$ & $101.5(24.6)$ \\
\hline \multicolumn{2}{|l|}{ CKD stage $[\mathrm{n}, \%]$} \\
\hline Stage 1 & $103(73.0)$ \\
\hline Stage 2 & $32(22.7)$ \\
\hline Stage 3 & $4(2.8)$ \\
\hline Stage 4 & $2(1.4)$ \\
\hline 24-h proteinuria [g/24 h, median (IQR)] & $4.6(2.8,6.4)$ \\
\hline Hemoglobin $[\mathrm{g} / \mathrm{l}$, mean $(\mathrm{SD})]$ & $132.4(15.1)$ \\
\hline Fibrinogen $[\mathrm{g} / \mathrm{l}$, mean $(\mathrm{SD})]$ & $5.1(1.4)$ \\
\hline $\begin{array}{l}\text { Serum anti-PLA }{ }_{2} \mathrm{R} \text { antibody }[\mathrm{Ru} / \mathrm{ml} \text {, } \\
\text { median }(\mathrm{IQR})]\end{array}$ & $171.1(11.6,561.8)$ \\
\hline Hypertension $(\mathrm{n}, \%)$ & $82(58.2)$ \\
\hline $\mathrm{SBP}[\mathrm{mmHg}$, mean $(\mathrm{SD})]$ & $135.0(21.9)$ \\
\hline $\mathrm{DBP}[\mathrm{mmHg}$, mean $(\mathrm{SD})]$ & $80.3(12.9)$ \\
\hline
\end{tabular}

B, Characteristics during follow-up

\begin{tabular}{lc}
\hline Characteristic & Value \\
\hline Follow-up [months, median (IQR)] & $30(21.0,45.0)$ \\
Corticosteroids (n, \%) & $115(81.6)$ \\
Immunosuppressants (n, \%) & \\
NO & $38(27.0)$ \\
CTX & $28(19.9)$ \\
CNI & $46(32.6)$ \\
Others & $29(20.6)$ \\
Remission (n, \%) & \\
CR & $41(29.1)$ \\
PR & $80(56.7)$ \\
NR & $20(14.2)$ \\
Relapse (n, \%) & $23(19.0)$ \\
ACEI (n, \%) & $48(34.0)$ \\
ARB (n, \%) & $130(92.2)$ \\
Endpoints (n, \%) & $18(12.8)$ \\
ESRD (n, \%) & $6(4.3 \%)$ \\
Death (n, \%) & $1(0.7 \%)$ \\
\hline
\end{tabular}

$\mathrm{SD}$, standard deviation; HDL-C, high-density lipoprotein cholesterol; LDL-C, low-density lipoprotein cholesterol; eGFR, estimated glomerular filtration rate; CKD, chronic kidney disease; IQR, interquartile range; SBP, systolic blood pressure; DBP, diastolic blood pressure; CR, complete remission; PR, partial remission; NR, no remission; ACEI, angiotensin-converting enzyme inhibitor; ARB, angiotensin receptor blocker; ESRD, end-stage renal disease.
Table II. Differences in pathological lesions between patients with and without poor renal outcomes.

\begin{tabular}{lccc}
\hline & \multicolumn{2}{c}{ Poor renal outcome } & \\
\cline { 2 - 3 } Characteristics & No & Yes & P-value \\
\hline Cases (n) & 123 & 18 & \\
MN stage (n, \%) & & & 0.22 \\
Stage I & $51(42.1)$ & $8(47.1)$ & \\
Stage II & $66(54.5)$ & $7(41.2)$ & \\
Stage III & $4(3.3)$ & $2(11.8)$ & \\
Chronic tubulointerstitial & & & 0.01 \\
injury (n, \%) & & & \\
Grade 0 & $71(57.7)$ & $4(22.2)$ & \\
Grade 1 & $39(31.7)$ & $9(50.0)$ & \\
Grade 2 & $13(10.6)$ & $5(27.8)$ & \\
IgG deposition (n, \%) & & & \\
IgG1 (n, \%) & $82(90.1)$ & $14(87.5)$ & 1.00 \\
IgG2 (n, \%) & $3(3.3)$ & $1(6.2)$ & 1.00 \\
IgG3 (n, \%) & $4(4.4)$ & $0(0.0)$ & 0.89 \\
IgG4 (n, \%) & $87(95.6)$ & $14(87.5)$ & 0.48 \\
C3 deposition (n, \%) & $104(85.2)$ & $15(88.2)$ & 1.00 \\
\hline
\end{tabular}

agents to reduce urinary protein extraction and/or control BP. Eighteen $(12.8 \%)$ patients eventually progressed to the primary endpoints, 6 (4.3\%) of whom developed ESRD, and one patient died due to a massive cerebral infarction mainly caused by nephrotic syndrome during the follow-up. These results are listed in Table I.

Comparing differences in pathological lesions in patients with different renal outcomes. Cases with different renal outcomes were divided into two groups: Those with poor renal outcome (Yes) and those without poor renal outcome (No). The results are listed in Table II. In the present study cohort, the majority of patients had stage 1-2 membranous lesions, and no significant difference was found between the Yes and No groups. However, a significant difference was found for chronic tubulointerstitial injury $(\mathrm{P}=0.01)$, with the Yes group showing an association with more severe tubulointerstitial injury in comparison with the No group. No significant difference in the intensity of immunofluorescence staining between the Yes and No groups was found. All cases in the present cohort displayed diffuse global granular polyclonal IgG deposition along the glomerular capillary wall, and the main subtypes of IgG were IgG1 and IgG4. Approximately $85-88 \%$ of the cases had complementary C3 diffuse global granular deposition along the glomerular capillary loop. None of these cases had Clq deposition.

Relationship between clinical and pathological variables and poor renal outcomes. The relationship between poor renal outcomes and clinical or pathological variables at the time of renal biopsy was assessed by univariate Cox proportional hazard regression (Table III). Serum uric acid [hazard ratio (HR), 1.44; 95\% confidence interval (CI), 1.09-1.91; P-value $=0.01]$, eGFR (HR, 0.97; 95\% CI, 0.95-0.99; P-value=0.001), 24-h 
Table III. Relationships between poor renal outcomes and clinical and pathological variables.

\begin{tabular}{|c|c|c|c|c|}
\hline \multirow[b]{2}{*}{ Characteristics } & \multicolumn{2}{|c|}{ Univariate model } & \multicolumn{2}{|c|}{ Multivariate model } \\
\hline & $\mathrm{HR}(95 \% \mathrm{CI})$ & P-value & HR $(95 \% \mathrm{CI})$ & P-value \\
\hline Sex (female) & $0.75(0.29-1.96)$ & 0.56 & & \\
\hline Age (years) & $1.03(0.99-1.07)$ & 0.19 & & \\
\hline Serum albumin $(g / l)$ & $0.93(0.84-1.01)$ & 0.10 & $1.00(0.90-1.11)$ & 0.99 \\
\hline Total cholesterol (mmol/l) & $1.12(0.9-1.38)$ & 0.31 & & \\
\hline Triglycerides (mmol/l) & $1.23(0.91-1.65)$ & 0.18 & & \\
\hline HDL-C (mmol/l) & $0.35(0.09-1.41)$ & 0.14 & & \\
\hline LDL-C (mmol/l) & $1.09(0.83-1.45)$ & 0.53 & & \\
\hline Serum uric acid $(\mathrm{mg} / \mathrm{dl})$ & $1.44(1.09-1.91)$ & 0.01 & $1.31(0.95-1.79)$ & 0.10 \\
\hline eGFR (ml/min/1.73 m²) & $0.97(0.95-0.99)$ & 0.001 & $0.99(0.97-1.02)$ & 0.58 \\
\hline 24-h proteinuria $(\mathrm{g} / 24 \mathrm{~h})$ & $1.22(1.11-1.33)$ & $<0.001$ & $1.23(1.08-1.41)$ & 0.002 \\
\hline Hemoglobin $(\mathrm{g} / \mathrm{l})$ & $0.99(0.95-1.02)$ & 0.48 & & \\
\hline Fibrinogen $(\mathrm{g} / \mathrm{l})$ & $1.28(0.89-1.83)$ & 0.18 & & \\
\hline Hypertension (Yes) & $10.78(1.43-81.08)$ & 0.02 & $4.05(0.49-33.75)$ & 0.20 \\
\hline $\mathrm{SBP}(\mathrm{mmHg})$ & $1.03(1.01-1.05)$ & 0.005 & & \\
\hline $\mathrm{DBP}(\mathrm{mmHg})$ & $1.02(0.99-1.05)$ & 0.24 & & \\
\hline Serum anti-PLA ${ }_{2} \mathrm{R}$ antibody $(\mathrm{Ru} / \mathrm{ml})$ & $0.99(0.98-1.01)$ & 0.31 & & \\
\hline \multicolumn{5}{|l|}{ Stage of membranous lesions } \\
\hline Stage I & 1 & - & & \\
\hline Stage II & $1.03(0.37-2.91)$ & 0.95 & & \\
\hline Stage III & $3.74(0.77-18.09)$ & 0.10 & & \\
\hline \multicolumn{5}{|l|}{ Chronic tubulointerstitial injury } \\
\hline Grade 0 & 1 & - & 1 & - \\
\hline Grade 1 & $6.01(1.61-22.42)$ & 0.008 & $4.84(1.22-19.24)$ & 0.03 \\
\hline Grade 2 & $10.91(2.48-48.00)$ & 0.002 & $5.38(0.95-30.42)$ & 0.06 \\
\hline Corticosteroids & $8.93(0.26-3.10)$ & 0.86 & & \\
\hline \multicolumn{5}{|l|}{ Immunosuppressants } \\
\hline NO & 1 & - & & \\
\hline CTX & $0.47(0.11-2.13)$ & 0.33 & & \\
\hline CNI & $0.86(0.16-4.76)$ & 0.86 & & \\
\hline Others & $2.39(0.73-7.84)$ & 0.15 & & \\
\hline
\end{tabular}

proteinuria (HR, 1.21; 95\% CI, 1.11-1.33; P-value <0.001), hypertension (HR, 10.78; 95\% CI, 1.43-81.08; P-value=0.02), systolic BP (HR, 1.03; 95\% CI, 1.01-1.05; P-value=0.005) and chronic tubulointerstitial injury [referred to as grade 0 , grade 1 (HR, 6.01; 95\% CI, 1.61-22.42; P-value $=0.008)]$ and grade 2 (HR, 10.91; 95\% CI 2.48-48.00; P-value $=0.002$ ) were significantly associated with poor renal outcomes. However, other variables, including the serum anti-PLA2R antibody level, showed no significant correlation with poor renal outcomes in the present study cohort. Covariates with P-values $<0.1$ were selected to build a multivariate Cox regression model, but systolic BP was removed considering the collinearity. The multivariate Cox regression model fit well for predicting a poor renal outcome with a high $\mathrm{C}$-index $(86 \%$; $95 \% \mathrm{CI}$, 78-94\%; P-value <0.001). Furthermore, according to the AIC, serum albumin level and eGFR were removed from the multivariate model, and four covariates (24-h proteinuria, serum uric acid, chronic tubulointerstitial injury and hypertension) were maintained to construct an optimized multivariate Cox regression model (Table IV). In particular, 24-h proteinuria (HR, 1.24; 95\% CI, 1.10-1.40; P-value <0.001) and chronic tubulointerstitial injury [referred to as grade 0; grade 1 (HR, 5.12; 95\% CI, 1.33-19.75; P-value $=0.02)$ or grade $2(\mathrm{HR}$, 6.43; 95\% CI, 1.35-30.59; P-value=0.02) were independent risk factors for a poor renal outcome. Therneau-Grambsch tests demonstrated that the four covariates in the optimized model were all independent of time (Table IV), which is a key assumption of the Cox regression. The $\mathrm{C}$-index of the model was $87 \%$ (95\% CI, 79-95\%; P-value <0.001) and no significant difference was found between the two multivariate Cox models $\left(\chi^{2}\right.$ test; P-value $\left.=0.86\right)$. A nomogram was plotted based on the optimized model to facilitate the calculation of the ETR (Fig. 1A).

Validation of the optimized model. A calibration plot was used to display the internal validity of the optimized model 
Table IV. Optimized prediction model.

\begin{tabular}{|c|c|c|c|c|}
\hline \multirow[b]{2}{*}{ Characteristics } & \multicolumn{2}{|c|}{ Results of regression } & \multicolumn{2}{|c|}{ Therneau-grambsch test } \\
\hline & $\mathrm{HR}(95 \% \mathrm{CI})$ & P-value & $\rho\left(\chi^{2}\right)$ & P-value \\
\hline 24-h proteinuria $(\mathrm{g} / 24 \mathrm{~h})$ & $1.24(1.10-1.40)$ & $<0.001$ & $-0.05(0.04)$ & 0.85 \\
\hline Serum uric acid (mg/dl) & $1.33(0.98-1.81)$ & 0.07 & $-0.28(1.13)$ & 0.29 \\
\hline Hypertension (Yes) & $4.31(0.53-35.35)$ & 0.17 & $-0.19(0.58)$ & 0.45 \\
\hline \multicolumn{5}{|c|}{ Chronic tubulointerstitial injury } \\
\hline Grade 0 & 1 & - & 1 & - \\
\hline Grade 1 & $5.12(1.33-19.75)$ & 0.02 & $0.21(0.89)$ & 0.35 \\
\hline Grade 2 & $6.43(1.35-30.59)$ & 0.02 & $0.13(0.31)$ & 0.58 \\
\hline
\end{tabular}

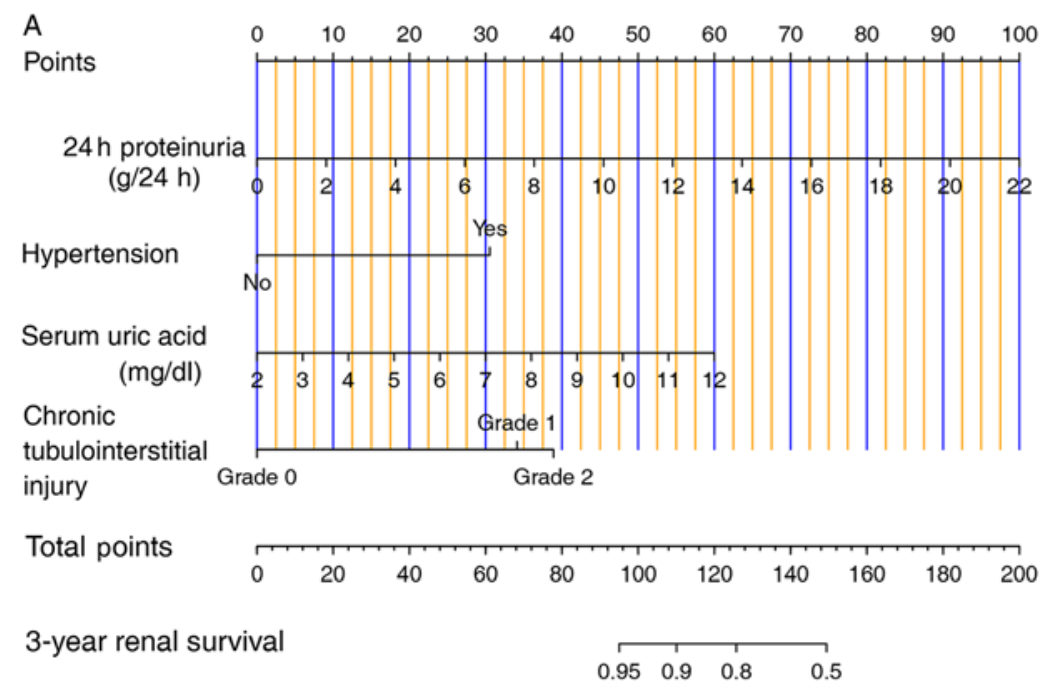

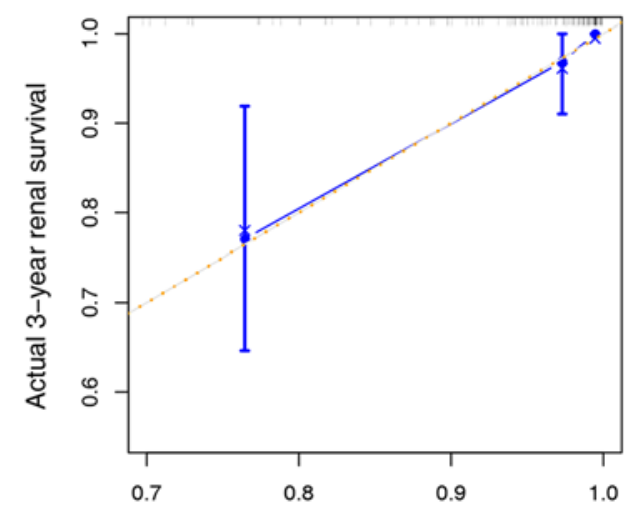

Nomogram-predicted 3-year renal survival

Figure 1. Nomogram of a model for estimation of the probability of renal survival and bootstrap calibration. (A) Nomogram of the optimized model. Points were assigned to parameters by drawing lines upward from the corresponding values to the 'Points' line. The sum of these points, plotted on the 'Total points' line, corresponds to the predicted three-year survival as the primary endpoint. (B) Calibration plot of the nomogram. The predictive line (solid line) overlaps well with the ideal line (dotted line), indicating that the predictive value approximates the actual value.

(Fig. 1B). The bootstrapping validation analysis illustrated that the optimized model showed good discrimination, with a high corrected C-index of $84 \%$. The ROC analysis of the optimized model showed an AUC of 0.83 (95\% CI, 0.73-0.92), and the specificity and sensitivity were $85 \%$ (95\% CI, 79-91\%) and $67 \%$ (95\% CI, 44-89\%), respectively. Based on the optimized model, the best cut-off value of the ETR was 0.87 (Fig. 2). Compared to the traditional risk factors of iMN, the AUCs were significantly decreased for 24-h proteinuria (AUC, 0.69; 95\% CI, 0.55-0.85) and serum uric acid (AUC, $0.68 ; 95 \%$ CI, 0.55-0.80) and slightly reduced for the eGFR (AUC, 0.70; 95\% CI, 0.56-0.85).

Changes in proteinuria and the eGFR at different levels of the ETR. The cases in the present study cohort were divided into four groups according to the quartiles of the ETR (Q1, 0.09-0.90; Q2, 0.90-0.98; Q3, 0.98-0.99 and Q4, 0.99-1.00; with boundary values divided into lower intervals), and the 24-h proteinuria levels corresponding to the ETR were calculated (Q1, 21.7-8.8 g; Q2, 8.8-4.0 g; Q3, 4.0-2.0 g and Q4, 2.0-0 g; with boundary values divided into lower intervals). Referring to the Toronto Risk Score risk classification (14), the four groups were classified into three groups: Q1, the high-risk group, Q2, the medium-risk group and Q3 and Q4, the low-risk group. The changes in proteinuria and the eGFR in different categories of the ETR are displayed in Fig. 3. Significant correlations were found between the ETR and changes in proteinuria or the eGFR (both P-values <0.001). The changes in proteinuria were not significantly different between the Q1 and Q2 groups [mean difference (MD), -0.9; 95\% CI, -2.10-0.30; P-value=0.21], but significant differences were found between the Q1 and Q3 groups (MD, -2.68; 95\% CI, -3.87- -1.50; P-value <0.001) and the Q1 and Q4 groups (MD, -2.80; 95\% CI, -3.99- -1.61; P-value $<0.001$ ). Additionally, the changes in the eGFR were significantly different between the Q1 group and the other groups: Q2 $(\mathrm{MD}=10.52,95 \%$ CI 1.64-19.39, $\mathrm{P}$-value $=0.01), \mathrm{Q} 3(\mathrm{MD}=20.01$, 95\% CI 11.21-28.81, P-value <0.001), and Q4 (MD=31.96, 95\% CI 23.16-40.76, P-value <0.001).

\section{Discussion}

Given the toxicity of immunosuppressive therapies and the natural course of iMN, early prediction of renal outcomes is 


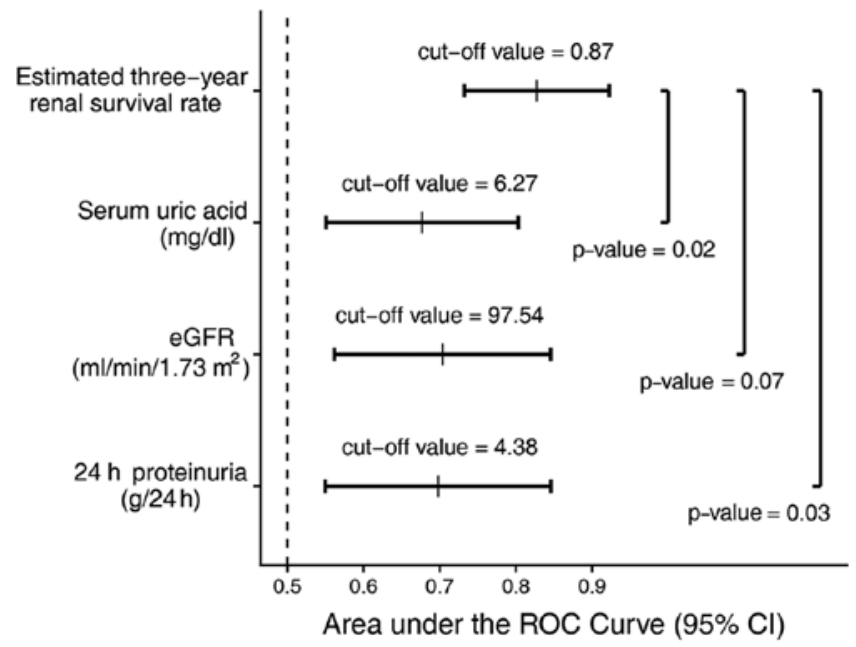

Figure 2. Comparison of the differences in the AUC between the ETR and traditional risk factors (24-h proteinuria, eGFR, and serum uric acid) for the identification of poor renal outcomes. AUC, area under the ROC curve; CI, confidence interval; eGFR, estimated glomerular filtration rate; ROC, receiver operating characteristic.

critical for therapy selection (32). However, to the best of our knowledge, there is no nomogram for the early prediction of the renal outcomes among patients with iMN at present. The current study showed that 24-h proteinuria and chronic tubulointerstitial injury are independently associated with poor renal outcomes in patients with iMN, and a validated model and nomogram for the early prediction of renal outcomes based on an optimized multivariate Cox proportional hazard model incorporating four variables at the time of renal biopsy (24-h proteinuria, serum uric acid, chronic tubulointerstitial injury, and hypertension) was established.

Although the time at which immunosuppressive therapy is administered to patients with iMN is still controversial, aggressive immunosuppressant therapy has been confirmed to increase the remission rate of nephrotic syndrome and improve renal prognosis (6). In the present study cohort, $81.6 \%$ of patients received glucocorticoid therapy and $73 \%$ of patients received other immunologic agents. Among the cohort, 85.8\% of patients achieved remission, which is significantly higher than the proportion of spontaneous remission $(17,33)$.

Previous research has shown that male sex, older than 60 years, hypertension, nephrotic-range proteinuria and decreased eGFR are associated with a poor prognosis $(8,16,33-35)$. The results of the present study cohort also indicated that the $24-\mathrm{h}$ proteinuria level at the time of renal biopsy was an independent predictor for a poor renal outcome. Renal histology is usually required to diagnose $\mathrm{MN}$, and most studies have suggested that pathological renal lesions other than chronic tubulointerstitial injury are not useful for predicting renal outcomes, which is consistent with the present results $(36,37)$. In the present model, chronic tubulointerstitial injury was also an independent predictor for poor renal outcomes.

Unexpectedly, eGFR was removed by the AIC analysis, but serum uric acid and hypertension were maintained. Although the role of uric acid in the progression of CKD is controversial, increasing evidence has indicated that uric acid
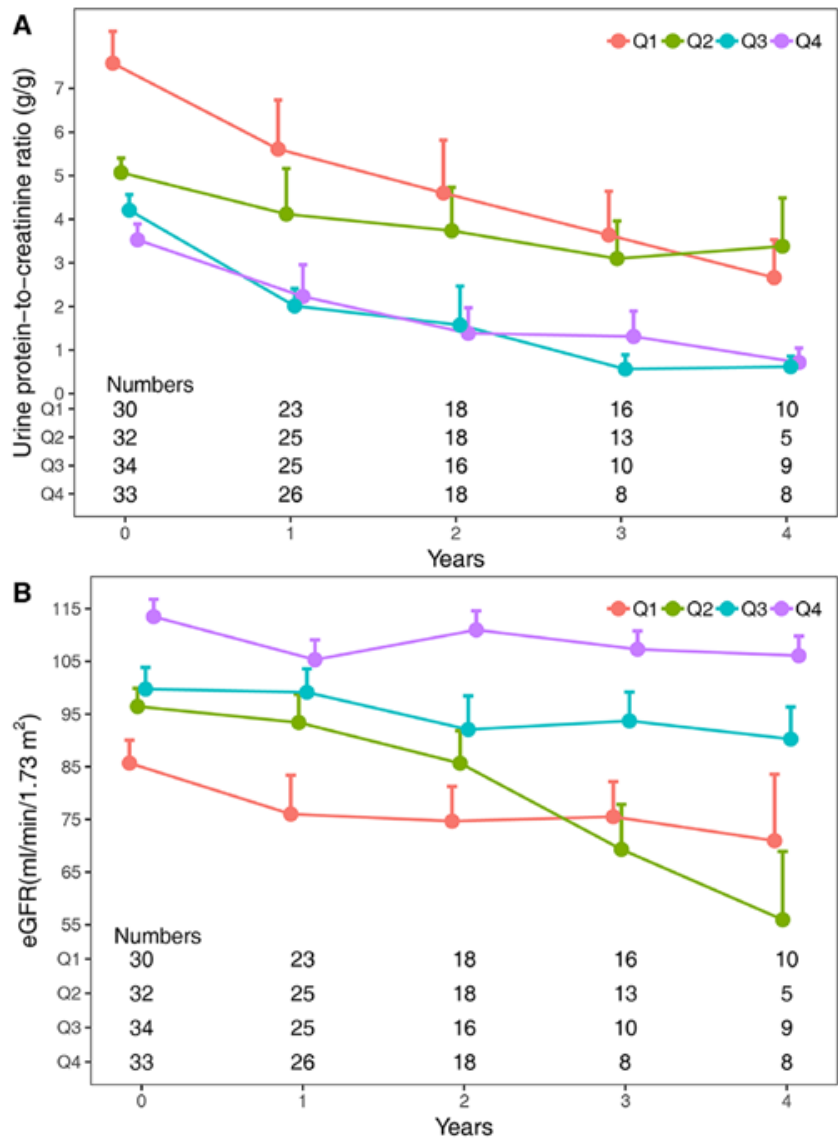

Figure 3. Trends of proteinuria and the eGFR for different ETRs. Cases were divided into four groups according to the quartiles of the ETR. (A) Changes in the urine protein-to-creatinine ratio. (B) Changes in the eGFR during the follow-up. Points and bars represent the mean and standard error, respectively. eGFR, estimated glomerular filtration rate; ETR, estimated three-year renal survival rate.

plays a considerable role in the progression of $\operatorname{CKD}(38,39)$. Approximately $10-67 \%$ of patients have hypertension at the onset of MN (37,40). In the present study cohort, 58.2\% of the patients had hypertension at the time of renal biopsy. Other studies have also demonstrated that the presence of hypertension at the onset of $\mathrm{MN}$ is a risk factor for poor renal outcomes $(8,17,18)$.

Compared to other studies, the present model had some advantages $(14,34)$. The four variables of the present model were confirmed to fit the assumption of Cox regression analysis and can be obtained easily at the time of renal biopsy. Furthermore, internal validation demonstrated that the model was robust for predicting the ETR and was significantly preferred over individuals' variables for the identification of poor renal outcomes. According to the ROC analysis, the specificity and sensitivity of the present model for the identification of poor renal outcomes were 85 and $67 \%$, respectively, and the best cut-off value of the ETR was 0.87 . In other words, patients with an ETR $<0.87$ likely progress to a poor renal outcome.

The present model indicated that the patients divided into the Q1 and Q2 groups would show more severe proteinuria and rapid progression of renal function decline, demonstrating that the model effectively predicted renal outcomes at an early stage. However, the changes in proteinuria and the 
eGFR were significantly different in the Q2 and Q3 groups, suggesting that the model may be useful for the early identification of medium-risk patients. Furthermore, after two years of follow-up, the patients in the Q2 group more quickly developed a decreased eGFR, indicating that more aggressive immunosuppressive treatment is warranted for these patients.

There are several limitations to the present study. First, this was a small retrospective study, which may have caused bias in the results. Second, few cases reached the endpoint due to the nature of the cases that were included in the present study and the duration of the follow-up. Although a variety of rigorous statistical analyses were used to verify the results, the stability of the model may have been affected. Third, most of the enrolled patients had received various immunosuppressive therapies during the follow-up that may have shifted the survival curve. Finally, the enrolled patients were from a single center and were of the same ethnicity, which may affect the scalability of the model.

In conclusion, the present study suggested a new optimized Cox regression model for the early prediction of renal outcomes in patients with iMN and developed a nomogram for the convenient calculation of the ETR. Patients with an ETR of $<0.87$ may require early immunosuppressive treatment. In addition, more attention should be directed toward patients with an ETR of 0.87-0.98 during follow-up.

\section{Acknowledgements}

The authors would like to thank their colleagues, Dr Xiaokai Ding, Dr Bo Chen, Dr Lvying Qiu, Dr Yu Zheng, Dr Xiaohan You, Dr Jianna Zhang, at the Department of Nephrology, The First and Second Affiliated Hospitals of Wenzhou Medical University, for their hard work of performing routine follow-up.

\section{Funding}

The present study was supported by the Medical Health Science and Technology Project of Zhejiang Provincial Health Commission (grant no. 2020377868) and the Fund for Lin He's Academician Workstation of New Medicine and Clinical Translation (grant no. 18331210).

\section{Availability of data and materials}

The datasets used and/or analyzed during the current study are available from the corresponding author on reasonable request.

\section{Authors' contributions}

JZ and SFP analyzed and interpreted the patient data regarding iMN. ZYL and LWJ collected and processed the clinical data. MP and DL reviewed and scored kidney histopathology. ZHZ and MP conceived and designed the current study. JZ, ZHZ, and MP were major contributors in writing the manuscript. All authors read and approved the final manuscript.

\section{Ethics approval and consent to participate}

The present study was performed after obtaining written informed consent from all patients and approved by the
Ethics Committee of the Second Affiliated Hospital \&Yuying Children's Hospital of Wenzhou Medical University (approval no. LCKY2019-217).

\section{Patient consent for publication}

Not applicable.

\section{Competing interests}

The authors declare that they have no competing interests.

\section{References}

1. Ronco P and Debiec H: Pathophysiological advances in membranous nephropathy: Time for a shift in patient's care. Lancet 385 : 1983-1992, 2015.

2. Cattran DC and Brenchley PE: Membranous nephropathy: Integrating basic science into improved clinical management. Kidney Int 91: 566-574, 2017.

3. Couser WG: Primary membranous nephropathy. Clin J Am Soc Nephrol 12: 983-997, 2017.

4. Beck LH Jr, Bonegio RG, Lambeau G, Beck DM, Powell DW, Cummins TD, Klein JB and Salant DJ: M-type phospholipase A2 receptor as target antigen in idiopathic membranous nephropathy. N Engl J Med 361: 11-21, 2009.

5. Tomas NM, Beck LH Jr, Meyer-Schwesinger C, Seitz-Polski B, Ma H, Zahner G, Dolla G, Hoxha E, Helmchen U, Dabert-Gay AS, et al: Thrombospondin type-1 domain-containing 7A in idiopathic membranous nephropathy. N Engl J Med 371: 2277-2287, 2014.

6. Ponticelli C, Zucchelli P, Passerini P, Cesana B, Locatelli F, Pasquali S, Sasdelli M, Redaelli B, Grassi C, Pozzi C, et al: A 10-year follow-up of a randomized study with methylprednisolone and chlorambucil in membranous nephropathy. Kidney Int 48: 1600-1604, 1995.

7. Hladunewich MA, Troyanov S, Calafati J and Cattran DC; Metropolitan Toronto Glomerulonephritis Registry: The natural history of the non-nephrotic membranous nephropathy patient. Clin J Am Soc Nephrol 4: 1417-1422, 2009.

8. Zuo K, Wu Y, Li SJ, Xu F, Zeng CH and Liu ZH: Long-term outcome and prognostic factors of idiopathic membranous nephropathy in the Chinese population. Clin Nephrol 79: 445-453, 2013.

9. van den Brand JA, Hofstra JM and Wetzels JF: Low-molecular-weight proteins as prognostic markers in idiopathic membranous nephropathy. Clin J Am Soc Nephrol 6: 2846-2853, 2011.

10. Ruggenenti P, Fervenza FC and Remuzzi G: Treatment of membranous nephropathy: Time for a paradigm shift. Nat Rev Nephrol 13: 563-579, 2017.

11. Segal PE and Choi MJ: Recent advances and prognosis in idiopathic membranous nephropathy. Adv Chronic Kidney Dis 19: 114-119, 2012.

12. Waldman M and Austin HA III: Controversies in the treatment of idiopathic membranous nephropathy. Nat Rev Nephrol 5: 469-479, 2009.

13. van den Brand JA, Hofstra JM and Wetzels JF: Prognostic value of risk score and urinary markers in idiopathic membranous nephropathy. Clin J Am Soc Nephrol 7: 1242-1248, 2012.

14. Cattran DC, Pei Y, Greenwood CM, Ponticelli C, Passerini P and Honkanen E: Validation of a predictive model of idiopathic membranous nephropathy: Its clinical and research implications. Kidney Int 51: 901-907, 1997.

15. Pei Y, Cattran D and Greenwood C: Predicting chronic renal insufficiency in idiopathic membranous glomerulonephritis. Kidney Int 42: 960-966, 1992.

16. Shiiki H, Saito T, Nishitani Y, Mitarai T, Yorioka N, Yoshimura A, Yokoyama H, Nishi S, Tomino Y, Kurokawa K, et al: Prognosis and risk factors for idiopathic membranous nephropathy with nephrotic syndrome in Japan. Kidney Int 65: 1400-1407, 2004.

17. Schieppati A, Mosconi L, Perna A, Mecca G, Bertani T, Garattini S and Remuzzi G: Prognosis of untreated patients with idiopathic membranous nephropathy. N Engl J Med 329: 85-89, 1993. 
18. Chembo CL, Marshall MR, Williams LC, Walker RJ, Lynn KL, Irvine $\mathrm{J}$ and Pilmore HL: Long-term outcomes for primary glomerulonephritis: New Zealand glomerulonephritis study. Nephrology (Carlton) 20: 899-907, 2015.

19. Dähnrich C, Komorowski L, Probst C, Seitz-Polski B, Esnault V, Wetzels JF, Hofstra JM, Hoxha E, Stahl RA, Lambeau G, et al: Development of a standardized ELISA for the determination of autoantibodies against human M-type phospholipase A2 receptor in primary membranous nephropathy. Clin Chim Acta 421: 213-218, 2013

20. Foundation NK: KDIGO clinical practice guideline for glomerulonephritis. Kidney Int Suppl 2: 142, 2012.

21. Matsushita K, Selvin E, Bash LD, Astor BC and Coresh J: Risk implications of the new CKD epidemiology collaboration (CKD-EPI) equation compared with the MDRD study equation for estimated GFR: The atherosclerosis risk in communities (ARIC) study. Am J Kidney Dis 55: 648-659, 2010.

22. Brien EO, Atkins N and Malley KO: Defining normal ambulatory blood pressure. Am J Hypertens 6: 201S-206S, 1993.

23. Fogo AB, Lusco MA, Najafian B and Alpers CE: AJKD atlas of renal pathology: Membranous nephropathy. Am J Kidney Dis 66: e15-e17, 2015.

24. Ehrenreich T, Porush JG, Churg J, Garfinkel L, Glabman S, Goldstein MH, Grishman E and Yunis SL: Treatment of idiopathic membranous nephropathy. N Engl J Med 295: 741-746, 1976.

25. Working Group of the International IgA Nephropathy Network and the Renal Pathology Society, Cattran DC, Coppo R, Cook HT, Feehally J, Roberts IS, Troyanov S, Alpers CE, Amore A, Barratt J, et al: The Oxford classification of $\operatorname{IgA}$ nephropathy: rationale, clinicopathological correlations, and classification. Kidney Int 76: 534-545, 2009.

26. Harrell FE Jr: Rms: Regression modeling strategies. R package version 5.1-0, Journal, 2017.

27. Therneau TM and Grambsch PM: Modeling survival data: Extending the Cox model. Springer Science \& Business Media, 2013.

28. Akaike $\mathrm{H}$ : Information theory and an extension of the maximum likelihood principle. In: Selected papers of hirotugu akaike. New York, Springer, pp199-213, 1998.

29. Grambsch PM and Therneau TM: Proportional hazards tests and diagnostics based on weighted residuals. Biometrika 81: 515-526, 1994.
30. Harrell FE Jr: Dupont wefC and others. m: Hmisc: Harrell Miscellaneous. R package version 4.0-3, Journal, 2017.

31. Team RC: R: A language and environment for statistical computing. Journal, 2017.

32. Fervenza FC, Sethi S and Specks U: Idiopathic membranous nephropathy: Diagnosis and treatment. Clin J Am Soc Nephrol 3: 905-919, 2008.

33. Polanco N, Gutiérrez E, Covarsí A, Ariza F, Carreño A, Vigil A Baltar J, Fernández-Fresnedo G, Martín C, Pons S, et al: Spontaneous remission of nephrotic syndrome in idiopathic membranous nephropathy. J Am Soc Nephrol 21: 697-704, 2010.

34. Zhang XD, Cui Z, Zhang MF, Wang J, Zhang YM, Qu Z, Wang X, Huang J, Wang F, Meng LQ, et al: Clinical implications of pathological features of primary membranous nephropathy. BMC Nephrol 19: 215, 2018.

35. Chen Y, Tang L, Feng Z, Cao X, Sun X, Liu M, Liu S, Zhang X, Li P, Wei R, et al: Pathological predictors of renal outcomes in nephrotic idiopathic membranous nephropathy with decreased renal function. J Nephrol 27: 307-316, 2014.

36. Horvatic I, Ljubanovic DG, Bulimbasic S, Knotek M, Prkacin I, Tisljar M and Galesic K: Prognostic significance of glomerular and tubulointerstitial morphometry in idiopathic membranous nephropathy. Pathol Res Pract 208: 662-667, 2012.

37. Reichert LJ, Koene RA and Wetzels JF: Prognostic factors in idiopathic membranous nephropathy. Am J Kidney Dis 31: 1-11, 1998.

38. Mwasongwe SE, Fülöp T, Katz R, Musani SK, Sims M, Correa A, Flessner MF and Young BA: Relation of uric acid level to rapid kidney function decline and development of kidney disease: The Jackson heart study. J Clin Hypertens (Greenwich) 20: 775-783, 2018.

39. Iseki K: Significance of hyperuricemia among community-based screening participants. Contrib Nephrol 192: 41-47, 2018.

40. Waldman M, Beck LH Jr, Braun M, Wilkins K, Balow JE and Austin HA III: Membranous nephropathy: Pilot study of a novel regimen combining cyclosporine and rituximab. Kidney Int Rep 1: 73-84, 2016.

This work is licensed under a Creative Commons Attribution-NonCommercial-NoDerivatives 4.0 International (CC BY-NC-ND 4.0) License. 